\title{
Infants born to mothers with IBD present with altered gut microbiome that transfers abnormalities of the adaptive immune system to germ-free mice
}

\author{
Joana Torres (10 1,2 Jianzhong $\mathrm{Hu}_{1}{ }^{3}$ Akihiro Seki, ${ }^{4}$ Caroline Eisele, ${ }_{1}^{3}$ Nilendra Nair, ${ }_{1}^{3}$ \\ Ruiqi Huang, ${ }_{1}^{5}$ Leonid Tarassishin, ${ }^{3}$ Bindia Jharap, ${ }^{6}$ Justin Cote-Daigneault, ${ }^{7}$ \\ Qixing Mao, 3,8 Ilaria Mogno, ${ }^{3}$ Graham J Britton, ${ }^{3,9}$ Mathieu Uzzan (1) , ${ }^{4}$ \\ Ching-Lynn Chen, ${ }^{10}$ Asher Kornbluth, ${ }^{11}$ James George, ${ }^{1}$ Peter Legnani, ${ }^{1}$ Elana Maser, ${ }^{1}$ \\ Holly Loudon, ${ }^{10}$ Joanne Stone, ${ }^{10}$ Marla Dubinsky, ${ }_{1}^{12}$ Jeremiah J Faith, ${ }^{3,4}$ \\ Jose C Clemente, ${ }^{3,4}$ Saurabh Mehandru, ${ }^{1,4}$ Jean-Frederic Colombel, ${ }^{1}$ Inga Peter ${ }^{3}$
}

\begin{abstract}
Additional material is published online only. To view please visit the journal online (http://dx.doi.org/10.1136/ gutjnl-2018-317855)
\end{abstract}

For numbered affiliations see end of article.

Correspondence to Dr Inga Peter, Mt Sinai School of Medicine, New York, NY 10029, USA; inga.peter@mssm.edu

Received 2 November 2018 Revised 19 March 2019 Accepted 9 April 2019 Published Online First 29 April 2019
Check for updates

(C) Author(s) (or their employer(s)) 2020. No commercial re-use. See rights and permissions. Published by BMJ.

To cite: Torres J, Hu J, Seki A, et al. Gut 2020;69:42-51.

\section{ABSTRACT}

Background and aims Prenatal and early life bacterial colonisation is thought to play a major role in shaping the immune system. Furthermore, accumulating evidence links early life exposures to the risk of developing IBD later in life. We aimed to assess the effect of maternal IBD on the composition of the microbiome during pregnancy and on the offspring's microbiome.

Methods We prospectively examined the diversity and taxonomy of the microbiome of pregnant women with and without IBD and their babies at multiple time points. We evaluated the role of maternal IBD diagnosis, the mode of delivery, antibiotic use and feeding behaviour on the microbiome composition during early life. To assess the effects of IBD-associated maternal and infant microbiota on the enteric immune system, we inoculated germ-free mice (GFM) with the respective stool and profiled adaptive and innate immune cell populations in the murine intestines. Results Pregnant women with IBD and their offspring presented with lower bacterial diversity and altered bacterial composition compared with control women and their babies. Maternal IBD was the main predictor of the microbiota diversity in the infant gut at 7, 14, 30, 60 and 90 days of life. Babies born to mothers with IBD demonstrated enrichment in Gammaproteobacteria and depletion in Bifidobacteria. Finally, GFM inoculated with third trimester IBD mother and 90-day infant stools showed significantly reduced microbial diversity and fewer class-switched memory B cells and regulatory T cells in the colon.

Conclusion Aberrant gut microbiota composition persists during pregnancy with IBD and alters the bacterial diversity and abundance in the infant stool. The dysbiotic microbiota triggered abnormal imprinting of the intestinal immune system in GFM.

\section{INTRODUCTION}

IBDs, comprising Crohn's disease (CD) and UC, result from aberrant mucosal immune responses to bacteria in genetically susceptible individuals. ${ }^{12}$ While family history is the strongest risk factor for developing IBD, the genetic loci associated with risk do not fully explain disease heritability. ${ }^{1}$ IBD

\section{Significance of this study}

What is already known on this subject?

- A positive family history remains the strongest risk factor for developing IBD; however, genetic susceptibility alone explains a small proportion of disease heritability.

- IBD affects women during their reproductive years, and around $25 \%$ become pregnant after their initial diagnosis. Human studies have shown that profound changes in the microbiome occur during pregnancy and that maternal health status and maternal microbiome play a role in shaping the microbiome and immune system of the neonate.

- The role of IBD in the maternal microbiome composition during pregnancy and its impact on the offspring's microbiome remains unclear.

affects women during their reproductive years and $25 \%$ become pregnant after diagnosis. Some studies have suggested that there may be a higher disease transmission rate from mothers with IBD as compared with fathers. ${ }^{3}{ }^{4}$ Dynamic changes in the diversity and abundance of the microbiome have been observed during pregnancy coinciding with adjustments in maternal immunity. ${ }^{5}$ While this balance is likely more complicated in pregnant women with IBD, it is largely unknown how pregnancy may affect the microbiota of patients with IBD. Moreover, increasing evidence suggests that maternal health status and microbiota during pregnancy may influence the infant's initial gut colonisation, ${ }^{6}$ playing a key role in shaping the immune system development. ${ }^{7}$ Of interest, early life exposures, many of which have an impact on microbiota development, have been linked to the future risk of developing IBD. ${ }^{8-11}$ However, no information exists on the impact of maternal IBD on the development of the offspring's gut microbiome. Therefore, we designed a study aimed at characterising 
Significance of this study

What are the new findings?

- Women with IBD maintain altered bacterial diversity and composition in their gut during pregnancy as compared with controls, with an enrichment in Gammaproteobacteria and a depletion in Bacteroidetes.

- Babies born to IBD mothers present with lower diversity and altered bacterial gut composition up to at least 3 months of life, characterised by an enrichment of Gammaproteobacteria and a depletion of Bifidobacteria, independent of other exposures, compared with babies born to control mothers.

- The altered microbiota induced changes within the adaptive immune system of the intestines in germ-free mice, suggesting that maternal IBD may have impact on the immune system of the offspring through microbial factors.

How might it impact on clinical practice in the foreseeable future?

- Targeting dysbiosis in pregnant women with IBD or during early infancy could foster the development of a healthy microbiome in the offspring, thereby reducing the future risk of IBD.

the microbiota of pregnant women with and without IBD and of their babies. We then compared the effect of the maternal and infant microbiome on the priming of the immune system of germ-free mice (GFM), a model to study interactions between the microbiome and the nascent immune system. ${ }^{12}$

\section{METHODS}

Study design and sample and data collection

Participants for this study were selected from the prospective Exploring MEChanisms of disease traNsmission In Utero through the Microbiome (MECONIUM) study. Pregnant women with IBD, and age and race/ethnicity-matched controls were recruited after signing an informed consent. Exclusion criteria included inability to provide consent, HIV/AIDS, autoimmune diseases, fetal chromosomal or structural abnormalities and active infection during pregnancy. After enrollment, pregnant women self-collected stool and saliva samples at each trimester. Following delivery, serial stool samples were collected from the infants at 7, 14, 30, 60 and 90 days (figure 1). We chose these early time points due to a relatively uniform feeding behaviour, as well as limited person-toperson contact and exposures associated with walking or crawling. Demographics and relevant clinical history were collected at the time of recruitment (baseline) and at each time point concurring with sampling. For women with IBD, disease type and surgical history were recorded. Additionally, at each trimester, medications (including vitamins and supplements), changes in medication and disease activity (Harvey Bradshaw index ${ }^{13}$ and physician's global assessment for $\mathrm{CD}$, and the Mayo score for $\mathrm{UC}^{14}$ ) were also registered. At delivery, gestational age, mode of delivery, complications and medications, baby's sex, birth weight and need for neonatal intensive care were recorded. Preterm birth was defined as delivery $<37$ weeks of gestation and low birth weight as a birth weight $\leq 2499$ grams. Information on infants' feeding behaviour (exclusive formula feeding, exclusive breastfeeding or mixed) and antibiotic use during follow-up was prospectively obtained at the time of each sampling. Study data were collected and managed

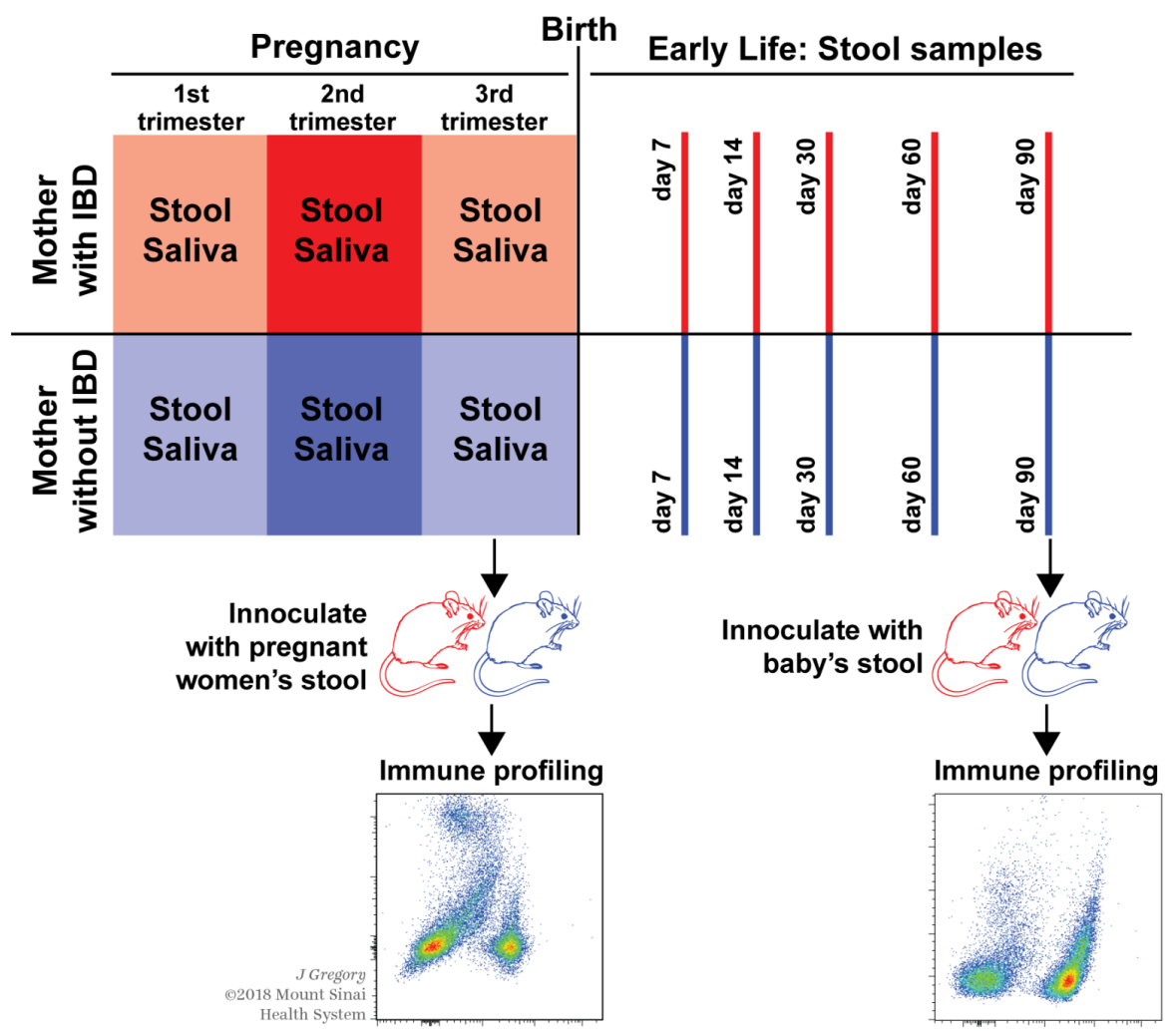

Figure 1 Study design: pregnant women with (upper panel) and without (lower panel) IBD recruited for the study collected a stool and saliva sample at each trimester, coupled with clinical and obstetric history. After delivery, their infants were followed up with serial stool samples and extensive health and exposure metadata at various time points. Stool from mother-baby pairs was selected and gavaged into wild-type germ-free mice aged 6-8 weeks to conduct immune phenotyping. 
using Research Electronic Data Capture electronic data capture tools. ${ }^{15}$

\section{Sample processing and 16S rRNA sequencing}

Total DNA was isolated from each specimen using a bead beating method with the PowerSoil DNA Isolation Kit (Mobio, Carlsbad, California, USA). After quantification by Qubit fluorometer, the DNA was amplified at the phylogenetically informative V3-V4 region of bacterial $16 \mathrm{~S}$ rRNA gene using dual-barcoded universal primer $347 \mathrm{~F} / 803 \mathrm{R}$ as described previously. ${ }^{16}$ The integrity of the amplicons was further verified by precast agarose gel electrophoresis. The resulting $\sim 460 \mathrm{bp}$ sized amplicons were pooled with equal molarity and then sequenced on the Illumina HiSeq2500 platform using the fast-mode pair-end 250 protocol. Sequentially, the $16 \mathrm{~S}$ reads were merged and filtered by size ( $>400 \mathrm{bp}$ ) and quality score ( $>$ Q30) using PANDAseq (PAired-eND Assembler for DNA sequences). ${ }^{17}$ The processed reads were further split by dual barcodes for each sample and then assigned to operational taxonomic units (OTUs) using open-reference OTU picking method with a default cut-off of 97\% similarities and taxonomic classification using the Quantitative Insights Into Microbial Ecology (QIIME) pipeline. ${ }^{18}$ Duplicate samples were included to assess sequencing reproducibility.

\section{Microbiome analysis of human samples}

We calculated the core diversity metrics at each time point and across body sites. Specifically, $\alpha$-diversity on rarefied tables was estimated using Faith's phylogenetic diversity, ${ }^{19}$ and significance between groups was determined using non-parametric tests. Microbial diversity between samples ( $\beta$-diversity) was evaluated with QIIME using unweighted UniFrac distances ${ }^{20}$ and visualised using multidimensional scaling plots. Multifactorial permutational analysis of variance (PERMANOVA) ${ }^{21}$ (Adonis in R package (vegan)) was used for each time point to test the association of maternal IBD status with the microbiome $\beta$-diversity while adjusting for covariates (feeding behaviour, mode of delivery, antibiotic exposure, preterm birth and baby's sex) and multiple hypothesis testing. The linear discriminant analysis effect size (LEfSe) algorithm ${ }^{22}$ was used to identify taxa associated with maternal IBD status. For infants, samples from all time points were pooled, and the differentially abundant features were ranked by effect size after adjusting for delivery mode due to significant effect of this variable on bacterial diversity. Taxa with $\geq 2$-fold differences by maternal IBD were selected. All statistical tests were two sided. To confirm robustness of our findings, MaAslin (Multivariate Association with Linear Models (MaAslin) was used in third trimester maternal samples for multivariate assessment of associations between taxa abundance and metadata, using default parameters. ${ }^{23}$ A linear mixed model was applied to the longitudinal offspring $16 \mathrm{~S}$ sequencing data to estimate the predictors of the most differential bacterial taxa identified in the LEfSe analysis while adjusting for constraint and time-varying covariates. Maternal IBD status, mode of delivery and time of sampling were treated as fixed effects, with a random intercept for each subject ID. Feeding behaviour and antibiotics exposure were entered as time-varying covariates. The model was fitted via restricted maximum likelihood approach with the correlation structure of autoregressive process of order 1.

\section{Microbiota transfer experiments in GFM} Donor selection and slurry preparation

To understand the functional consequences of altered microbiome in pregnant women and their offspring, stool samples from eight CD mother-baby pairs (16 individuals) and from three control mother-baby pairs (six individuals), for a total of
22 donors, were selected for microbiota transfer and gavaged into a total of $78 \mathrm{C} 57 \mathrm{Bl} / 6$ GFM. Donor selection included availability of paired stool samples from mother at third trimester and her baby at 90 days, full-term pregnancies and, for IBD mothers, inactive CD with similar medication patterns. Further details on donor features can be found in online supplementary table S1. Faecal samples were pulverised under liquid nitrogen; working under strict anaerobic conditions, samples were blended into a $40-60 \mathrm{mg} / \mathrm{mL}$ slurry in prereduced bacterial culture media. Infant stool samples collected on diaper material were suspended by repeated vortexing in $20 \mathrm{~mL}$ of prereduced bacterial culture media with $5 \mathrm{~mL}$ sterile $3 \mathrm{~mm}$ glass beads. Beads and diaper debris were removed by passing through a $100 \mu \mathrm{m}$ strainer. Faecal slurries were diluted 1:20 in bacterial culture media and stored at $-80^{\circ} \mathrm{C}$ in $15 \%$ glycerol until use.

\section{GFM gavage and sample collection}

$\mathrm{C} 57 \mathrm{Bl} / 6 \mathrm{GFM}$ were raised in gnotobiotic isolators and colonised at $6-8$ weeks of life by gavage with $200-300 \mu \mathrm{L}$ of recently thawed human faecal slurry; thereafter, mice were maintained outside the isolator in sterile filter-top cages and handled using strict aseptic techniques. ${ }^{24} 25$ Eight groups of mice (48 mice) were gavaged with the stool from CD mothers or their respective 3-month-old baby (2-5 GFM per donor). In addition, three groups of mice (30 mice) were gavaged with stool from non$\mathrm{CD}$, control pregnant women or their 3-month-old baby (3-5 GFM per donor). Five weeks after gavage, mice were euthanised by $\mathrm{CO}_{2}$; mesenteric lymph nodes (MLNs) and large intestine were harvested for immune profile analyses by flow cytometry. Additionally, murine stool samples were collected for 16S rRNA sequencing, following the protocol described above.

Single cell preparation from MLN, spleen and the intestinal lamina propria of mice

Initially, we examined the small intestinal lamina propria, colonic lamina propria, Peyer's patches and MLN separately (as components of the mucosal immune system including innate lymphoid cells (ILCs)). Additionally, splenocytes were evaluated to represent the systemic immune system. The MLN and spleen were harvested in RPMI media containing collagenase D (SigmaAldrich, final concentration $400 \mathrm{IU} / \mathrm{mL}$ ) and physically disrupted using a 25 -gauge syringe. Cell suspension was placed in an incubator at $37^{\circ} \mathrm{C}$ for $30 \mathrm{~min}$ followed by addition of $10 \mathrm{mM}$ EDTA (Invitrogen) and two rounds of washing with RPMI (Gibco).

Single cell suspension from the intestinal lamina propria was prepared as previously described with minor modification. ${ }^{26}$ Small intestines and colon were harvested and processed separately. Briefly, after Peyer's patches were removed from the small intestines. Mesenteric fat and the faecal contents were carefully removed from intestines, and the lumen was exposed using a longitudinal incision. The small and large intestinal tissues were washed with Hank's Balanced Salt Solution (HBSS) for three times, then cut into $1 \mathrm{~cm}$ pieces and incubated with $5 \mathrm{mM}$ EDTA (Invitrogen) in $\mathrm{HBSS}$ at $37^{\circ} \mathrm{C}$ for $20 \mathrm{~min}$ followed by washing with HBSS for three times. Next, the tissue was minced with scissors and transferred to conical tubes with $10 \mathrm{~mL}$ of complete RPMI containing $0.5 \mathrm{mg} / \mathrm{mL}$ of collagenase (Sigma-Aldrich) and incubated at $150 \mathrm{rpm}$ for $30 \mathrm{~min}$ at $37^{\circ} \mathrm{C}$. The single cell suspension was collected and passed through a $100 \mu \mathrm{m}$ and $40 \mu \mathrm{m}$ cell strainer and washed with RPMI twice.

After initial experiments, we focused on differences in immune populations within colonic tissues as representative of the intestinal immune system. 


\section{Flow cytometry analysis}

To prevent non-specific staining, the single cell suspension from MLN and colonic lamina propria was incubated with CD16/32 antibody (2.4G2, BioXcell). At the outset, we characterised T cell subsets, B cell subsets, dendritic cell (DC) subsets and ILCs using panels of fluorescent dyes conjugated with monoclonal antibodies (online supplementary table S2). LIVE/DEAD Fixable Aqua Dead Cell Stain Kit (Invitrogen) was used to determine the viability of cells. Foxp3 staining was performed after fixation and permeabilisation using the Foxp3/transcription factor staining buffer set (Invitrogen). Results from the colonic lamina propria were representative of changes in the mucosal immune system. Therefore, after initial experiments, we focused on analyses of the colonic lamina propria only in the GFM. Samples were acquired using LSR Fortessa (BD Bioscience), and data were analysed using FlowJo V.10 (FlowJo).

\section{ELISA for stool lgA}

Murine stool $(50 \mathrm{mg}$ ) was incubated in $500 \mu \mathrm{L}$ of PBS at room temperature for $10 \mathrm{~min}$, followed by vortexing for $5 \mathrm{~min}$ and bead-beating for $2 \mathrm{~min}$. This step was repeated twice, and the sample supernatants were collected after centrifugation. IgA ELISA was performed by using the Mouse IgA ELISA Quantitation Set (Bethyl Laboratories) according to the manufacturer's protocol. A MaxiSorp ELISA plate (Thermo Fisher Scientific) was precoated with $100 \mu \mathrm{L}$ of diluted purified IgA antibody in $0.1 \mathrm{M}$ carbonate buffer $(10 \mu \mathrm{l} / \mathrm{mL})$. After overnight incubation at $4^{\circ} \mathrm{C}$, the plate was washed with PBS and incubated with PBS containing $1 \%$ BSA for 1 hour at room temperature to prevent non-specific response. After washing, $100 \mu \mathrm{L}$ of 1:200 diluted stool supernatants were plated and incubated for 1 hour. The plates were washed again and $100 \mu \mathrm{L}$ of antimouse IgA-HRP
(1:40000 dilution) was added. After 1 hour incubation, $100 \mu \mathrm{L}$ of TMB substrate (BD Pharmingen) was added, followed by $100 \mu \mathrm{L} 1 \mathrm{M} \mathrm{H}_{2} \mathrm{SO} 4$ (as a stop solution). The absorbance (at $450 \mathrm{~nm}$ ) was measured using an ELISA reader.

\section{RESULTS}

\section{Characteristics of study participants}

We included the first 200 consecutive participants from the MECONIUM study: 121 pregnant women (40 with IBD), and 79 babies (26 born to mothers with IBD), resulting in a total of 73 mother-infant dyads. The demographic and clinical characteristics of pregnant women were evenly distributed by IBD status with regards to maternal age, gestational age at recruitment, pregnancy complications, use of assisted reproductive technologies and exposure to antibiotics during pregnancy or at delivery (table 1).

Mothers with IBD were more likely to be Jewish $(\mathrm{p}=0.008)$ and nulliparous $(p=0.024)$. Most pregnant women with IBD (70.0\%) presented with inactive disease. When occurred, flares were mostly mild. Patients with $\mathrm{CD}$ had a more frequent history of bowel resection $(p=0.02)$ compared with $\mathrm{UC}$ and demonstrated differences in medication (online supplementary table S3). Except for a higher proportion of male babies born to IBD mothers $(p=0.013$; table 1$)$, rates of preterm birth, low birth weight or exposure to antibiotics or probiotics after birth were similar across groups (table 1).

\section{Women with IBD present persistent dysbiosis during pregnancy}

After quality control, 619 samples with $>1000$ sequencing reads each were available for downstream analysis (total read counts:

Table 1 Demographic and clinical characteristics of study participants

\begin{tabular}{|c|c|c|c|}
\hline Characteristics & Mother with IBD & Control mother & $P$ value \\
\hline $\mathrm{N}$ & 40 & 81 & - \\
\hline Mean age at recruitment (year, mean $\pm S D$ ) & $32.9 \pm 4$ & $32.9 \pm 3.7$ & 0.35 \\
\hline Jewish ethnicity (\%) & 35 & 13.8 & 0.008 \\
\hline Nulliparous (\%) & 62.5 & 40.7 & 0.024 \\
\hline Exposure to antibiotics during pregnancy (\%) & 20.5 & 24.1 & 0.67 \\
\hline Peripartum antibiotics (\%) & 65.5 & 52.1 & 0.25 \\
\hline Use of assisted reproductive technology (\%) & 9.9 & 13.2 & 0.59 \\
\hline Gestational diabetes/type 1 DM (\%) & 3.9 & 5.7 & 0.67 \\
\hline Biologic drugs during pregnancy (\%) & 50 & - & - \\
\hline Thiopurines during pregnancy (\%) & 28 & - & - \\
\hline Prior surgery (\%) & 16 & - & - \\
\hline Disease remission during pregnancy (\%) & 70 & - & - \\
\hline Characteristics & Infants born to mothers with IBD & Infants born to control mothers & $P$ value \\
\hline N & 26 & 53 & \\
\hline Male gender (\%) & 69.2 & 39.6 & 0.013 \\
\hline Mean gestational age at delivery (weeks, mean \pm SD) & $39.5 \pm 1.08$ & $39.3 \pm 1.67$ & 0.36 \\
\hline Delivered by C-section (\%) & 46.2 & 30.2 & 0.16 \\
\hline Preterm birth (\%) & 5.7 & 0.0 & 0.55 \\
\hline Birth weight $(\mathrm{kg}$, mean $\pm \mathrm{SD})$ & $3.41 \pm 0.38$ & $3.29 \pm 0.52$ & 0.82 \\
\hline Low birth weight (\%) & 0 & 3.8 & 0.31 \\
\hline Exclusive breast feeding (\%) & 50 & 39.6 & 0.073 \\
\hline Exclusive formula (\%) & 15.4 & 3.7 & \\
\hline Mixed feeding (\%) & 34.6 & 56.6 & \\
\hline Exposure to antibiotics after birth (\%) & 3.8 & 9.4 & 0.66 \\
\hline Exposure to probiotics after birth (\%) & 11.5 & 13.2 & 0.83 \\
\hline
\end{tabular}


A
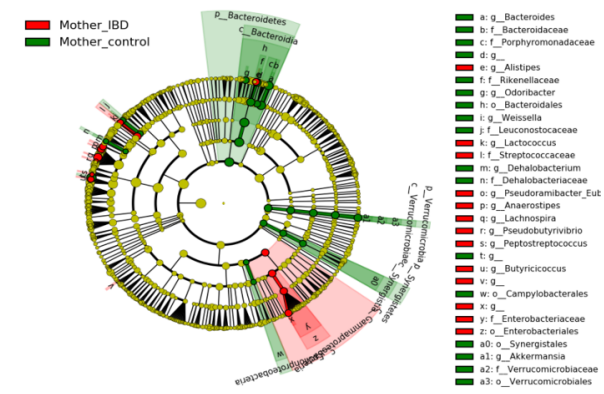

B
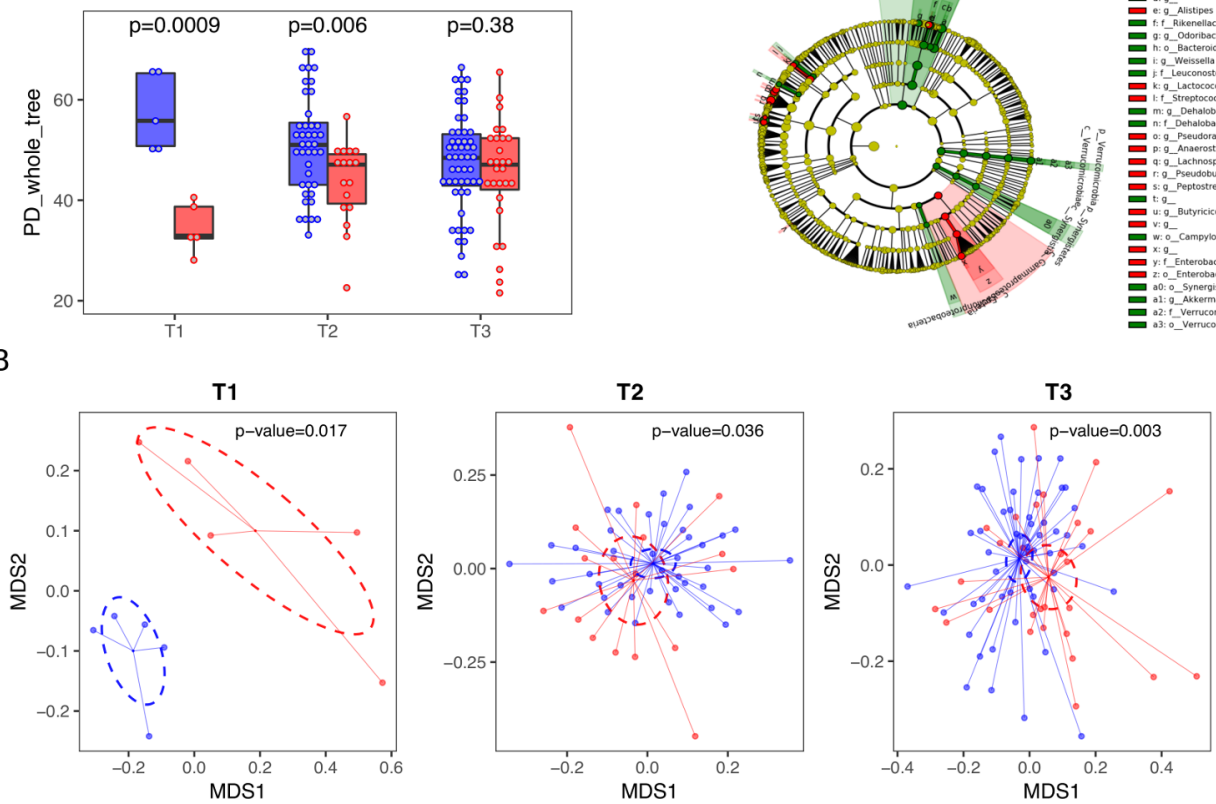

Figure 2 The gut microbiota composition of pregnant women by IBD diagnosis. (A) The boxplots show the mean and variance of the richness of the microbial community ( $\alpha$-diversity) between pregnant women with (red) and without (blue) IBD (PD_whole tree); significant differences were seen at the first (T1) $(p=0.005)$ and second trimester (T2) ( $p=0.01)$ but not in third trimester (T3). (B) Overall microbiota dissimilarities (B-diversity) between samples grouped by IBD status. Dissimilarities were measured using unweighted UniFrac distances and visualised using a non-metric multidimensional scaling plot. Significant differences in the B-diversity were observed at each trimester of pregnancy between pregnant women with (red) and without (green) IBD. (C) Differential microbial features between pregnant women with and without IBD as determined by linear discriminant analysis effect size analysis (linear discriminant analysis).

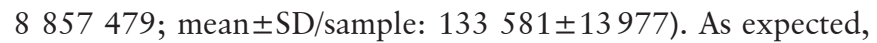
diversity was driven primarily by body sites (online supplementary figure S1a-d). Throughout pregnancy, women with IBD had lower gut $\alpha$-diversity than controls, with differences reaching statistical significance at the first (T1) and second (T2) trimesters of pregnancy $(\mathrm{p}=0.005$ and $\mathrm{p}=0.01$, respectively) but not at the third (T3) trimester (figure 2A). We observed opposite trends in the changes over time of the global $\alpha$-diversity throughout pregnancy by IBD status: while control women presented a continuous decrease from T1 to T3 (PD_whole tree in T1: $51.2 \pm 8.4$ vs T3: 45.0 \pm 8.6 , Wilcoxon $p=0.0 \overline{4} 7$ ), patients with IBD demonstrated a non-significant trend towards a slight increase (PD_whole tree in T1: 40.2 \pm 7.1 vs T3: $43.8 \pm 7.9, \mathrm{p}=0.17$ ) (figure 2A). PERMANOVA analysis based on the unweighted UniFrac distances revealed significantly different $\beta$-diversity of the gut microbiota by IBD status at each trimester $(p=0.017$, 0.036 and 0.003 for $\mathrm{T} 1, \mathrm{~T} 2$ and $\mathrm{T} 3$, respectively; figure $2 \mathrm{~B}$ ). Biomarker analysis comparing the relative abundances of the gut microbiome by IBD diagnosis showed that the major differences were driven by an enrichment of the Gammaproteobacteria class and a depletion of the Bacteroidetes phylum in mothers with IBD as compared with controls (figure 2C). In T3 alone, an unidentified genus from the Enterobacteriaceae family was enriched in pregnant women with IBD (unadjusted $\mathrm{p}=10^{-5}$, Bonferroni corrected $\mathrm{p}=0.02$ ); corroborating this finding, the LEfSe analysis showed 14 taxa under the Proteobacteria phyla to be enriched (online supplementary figure S2). Further analysis, using MaAslin, conducted in T3 samples only $(\mathrm{n}=80)$ and taking into account participant type, race, parity and exposure to antibiotics during pregnancy confirmed a significant enrichment in Enterobacteriaceae family associated with maternal IBD status (false discovery rate (FDR) adjustedp value: 0.03 ). No significant differences were observed between mothers exposed to thiopurines or biologicals during pregnancy or in those with active versus inactive disease (data not shown).

No significant differences in the $\alpha$-diversity or $\beta$-diversity of the oral microbial community were observed within subjects throughout pregnancy or between IBD patients and controls at any trimester (data not shown).

\section{Maternal IBD status predicts the diversity and taxonomy of the infant microbiome}

At the time of our analysis, 79 babies had been born and followed up for up to 3 months, with 306 high-quality stool samples available for analysis. Overall, the bacterial diversity changed over time in the pooled set of infant samples (online supplementary figure S3). The gut microbiome of infants born to IBD mothers demonstrated lower diversity and variation in the overall abundance compared with babies born to control mothers (figure 3). Specifically, significant differences in $\alpha$-diversity were detected at days 7,14 and 90 based on maternal IBD status (Wilcoxon $\mathrm{p}=0.05,0.03$ and 0.02 , respectively; figure $3 \mathrm{~A}$ ). Furthermore, while maternal IBD diagnosis was a significant predictor of baby's $\beta$-diversity (figure $3 \mathrm{~B}$ ) (univariate and multivariate PERMANOVA $\mathrm{p}<0.05$ for each time point), mode of delivery, antibiotic exposure, type of feeding and preterm birth were also significantly associated with the overall bacterial abundance at various time points (table 2). Stratified analysis in babies born vaginally and via $\mathrm{C}$-section showed that the maternal IBD diagnosis remained a significant predictor of microbiome diversity regardless of the delivery mode. Likewise, differences remained after excluding all samples from babies that were exclusively formula fed (online supplementary figure S4). 
A

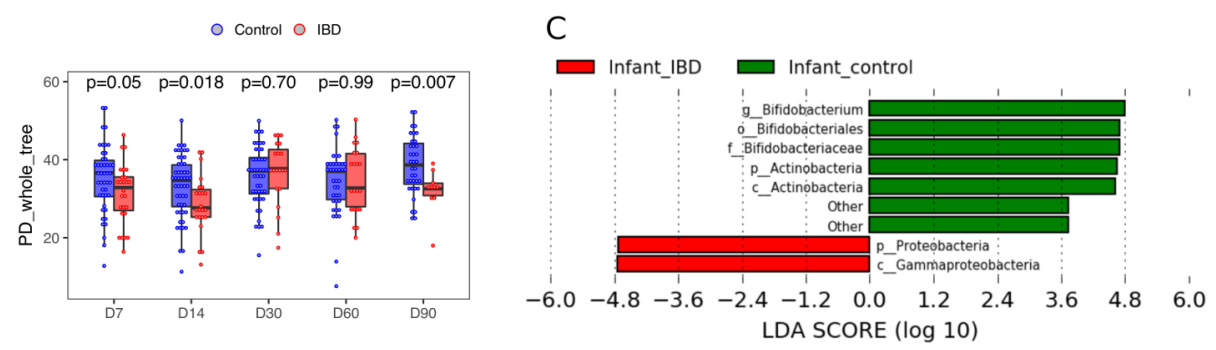

B
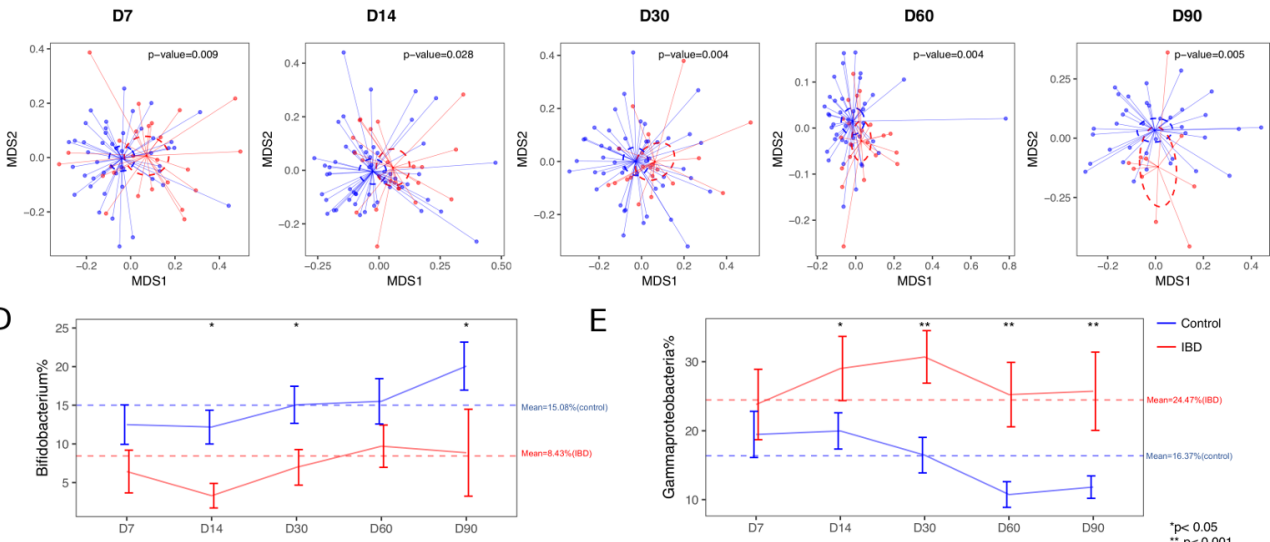

$\mathrm{E}$

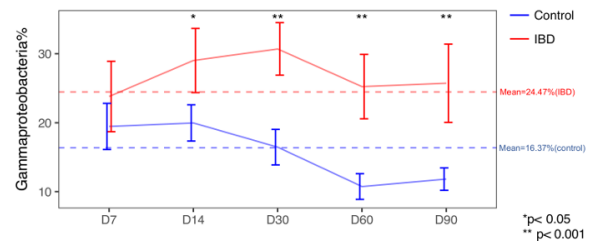

Figure 3 Microbiota profiles across multiple time points during early life by maternal IBD status. (A) The $\alpha$-diversity of the microbial community within samples obtained at different time points after delivery and compared between babies born to mothers with (red) and without (blue) IBD. The $\alpha$-diversity was measured using $P D \_$whole tree obtained in rarefied tables at 1000 sequences. (B) The $\beta$-diversity measured by unweighted UniFrac distances at different time points after delivery compared between babies by maternal IBD status. (C) Top discriminative bacteria between babies born to mothers with (red) and without (green) IBD as determined by the LEfSe analysis. ( $\mathrm{D}$ and $\mathrm{E}$ ) Representative differential bacterial taxa over time by maternal IBD status. Error bars indicate mean \pm SD. LDA, linear discriminant analysis; LEfSe, linear discriminant analysis effect size.

We then conducted LEfSe analysis across all time points while adjusting for the delivery mode, one of the major predictors of the early life microbiome. We detected that babies born to IBD mothers presented with an enrichment in the Gammaproteobacteria class bacteria and a reduction in the Bifidobacterium genus, as compared with those born to control mothers (figure 3C), and these differences persisted for the 3-month duration of the study (figure 3D,E). We next tested the impact of different exposures on the relative abundance of the differentially abundant taxa over time. We found that maternal IBD diagnosis and exclusive formula feeding were associated with a reduction in the relative abundance of beneficial Bifidobacterium genus over time (on the $\log$ scale 1.4 -fold reduction, $\mathrm{p}=0.007$ and 1.7 -fold reduction, $\mathrm{p}=0.048$, respectively; table 3 ). After adjustment for the covariates, maternal IBD status was the only significant predictor of the relative abundance of the colitogenic Gammaproteobacteria taxa over the first 3 months of life $(\mathrm{p}=0.002)$ (table 3 ).

\section{IBD status determines the composition of the adaptive immune system in GFM}

We first compared the overall bacterial abundance ( $\beta$-diversity) in the stool of GFM inoculated with the maternal and infant stools (design shown in figure 1 ) and detected significant differences by donor maternal IBD status $(p=0.0001$ and $p=0.003$, respectively, after adjustment for batch and repeated sampling; online supplementary figure $\mathrm{S} 5 \mathrm{a}-\mathrm{b})$.

Next, we characterised subsets of $\mathrm{T}$ cells, B cells, dendritic cells and ILCs in the intestines of GFM inoculated with maternal or infant stools. Non-IBD mother/baby stools and uninoculated

Table 2 Statistical significance for predictors of early life microbiota B-diversity by time point

\begin{tabular}{|c|c|c|c|c|c|c|c|c|c|c|}
\hline \multirow{2}{*}{$\begin{array}{l}\text { Time point } \\
\text { Covariates }\end{array}$} & \multicolumn{2}{|l|}{ Day 7} & \multicolumn{2}{|c|}{ Day 14} & \multicolumn{2}{|c|}{ Day 30} & \multicolumn{2}{|c|}{ Day 60} & \multicolumn{2}{|c|}{ Day 90} \\
\hline & Uni & Multi & Uni & Multi & Uni & Multi & Uni & Multi & Uni & Multi \\
\hline Maternal IBD status & 0.009 & 0.018 & 0.028 & 0.036 & 0.004 & 0.007 & 0.004 & 0.014 & 0.005 & 0.008 \\
\hline Delivery type & 0.001 & 0.001 & 0.001 & 0.001 & 0.010 & 0.008 & 0.056 & 0.120 & 0.050 & 0.029 \\
\hline Feeding & 0.045 & 0.070 & 0.002 & 0.002 & 0.028 & 0.064 & 0.110 & 0.400 & 0.001 & 0.090 \\
\hline Antibiotic exposure & 0.900 & 0.900 & 0.058 & 0.058 & 0.350 & 0.240 & 0.036 & 0.780 & 0.010 & 0.026 \\
\hline Sex & 0.100 & 0.110 & 0.213 & 0.137 & 0.300 & 0.500 & 0.700 & 1.000 & 0.018 & 0.084 \\
\hline Preterm birth & 0.690 & 0.560 & 0.015 & 0.098 & 0.500 & 0.160 & 0.280 & 0.410 & 0.003 & 0.001 \\
\hline
\end{tabular}

B-diversity was assessed using unweighted UniFrac distances from multifactorial PERMANOVA; number of permutations=999. Maternal IBD status is defined as case (IBD) or control. Delivery type is defined as vaginal delivery or caesarean section. Feeding type is defined as formula-exclusive feeding, exclusive breast feeding or mixed feeding (exposure to any formula after hospital discharge). Antibiotic exposure refers to exposure after birth only. Preterm birth was defined as birth occurring before 37 weeks of gestational age. Uni, univariate regression analysis, Multi, multivariate analysis adjusted for all listed covariates; PERMANOVA, permutational analysis of variance. 
Table 3 Results of the linear mixed regression model applied to the longitudinal changes in relative abundance of Bifidobacterium and Gammaproteobacteria during the first 3 months of life

\begin{tabular}{|c|c|c|c|c|c|c|}
\hline \multirow{2}{*}{$\begin{array}{l}\text { Differentially abundant taxa } \\
\text { Covariates }\end{array}$} & \multicolumn{3}{|l|}{ Bifidobacterium } & \multicolumn{3}{|c|}{ Gammaproteobacteria } \\
\hline & Estimated mean & SE & $P$ value & Estimated mean & SE & $P$ value \\
\hline (Intercept) & -4.63 & 0.39 & $<0.001$ & -3.33 & 0.24 & $<0.001$ \\
\hline IBD maternal status & -1.39 & 0.50 & 0.007 & 0.91 & 0.29 & 0.002 \\
\hline Time of sampling & 0.02 & 0.004 & $<0.001$ & -0.001 & 0.003 & 0.86 \\
\hline Born by C-section & -0.18 & 0.50 & 0.72 & 0.24 & 0.29 & 0.41 \\
\hline Exposure to antibiotics & -1.38 & 0.93 & 0.14 & 0.71 & 0.56 & 0.21 \\
\hline Feeding: formula exclusive & -1.75 & 0.88 & 0.048 & -0.78 & 0.54 & 0.15 \\
\hline Feeding: mixed & -0.45 & 0.39 & 0.25 & -0.50 & 0.26 & 0.05 \\
\hline
\end{tabular}

IBD status determines the composition of the adaptive immune system in GFM.

Repeated measurements included baby stool at 7, 14, 30, 60 and 90 days. Maternal IBD status, mode of delivery and time were treated as fixed effects, with a random intercept for each subject ID. Feeding behaviour and antibiotics exposure were entered as time-varying covariates. The model was fitted via restricted maximum likelihood approach with the correlation structure of autoregressive process of order 1.

C-section, caesarean section; GFM, germ-free mice.

GFM served as controls. Due to the good concordance in the results from the small intestinal lamina propria and the large intestinal lamina propria, we chose to focus on analyses of the colonic lamina propria only. We did not detect any significant differences between the spleen, Peyer's patches and MLN nor did we observe variation in the dendritic cells (DC) subsets or ILCs between GFM colonised with the stool of IBD pregnant women or their infants compared with no-IBD maternal or corresponding infant stool (data not shown).

Among lymphoid cells, while we did not detect differences in the total T cells as well as CD4 + T cells, there was a significant reduction in CD19+ B cells in the mesenteric lymph node of mice gavaged with the stool of mothers with IBD (online supplementary figure S6).

Additionally, we identified consistent and reproducible differences in the adaptive immune system. Specifically, in the colonic lamina propria of mice inoculated with stool of pregnant women with CD and their 3-month-old infants, the proportion of classswitched memory B cells (live $\mathrm{CD} 45^{+} \mathrm{CD} 19^{+} \mathrm{CD} 138^{-} \mathrm{IgD}^{-} \mathrm{IgM}^{-}$ cells; online supplementary figures S7 and S8) was significantly lower as compared with the mice inoculated with control maternal or 3-month infant stool, respectively (both $\mathrm{p}<0.005$ ) (figure 4a). Similar results were observed in MLN after inoculation with CD-exposed baby stool, but not CD maternal stool. Furthermore, among switched memory B cells, the homeostatic $\mathrm{IgA}+$ switched memory B cells were significantly reduced in the colonic LP of mice colonised with either CD mother or their baby stool $(p<0.01)$ but not in MLN (figure $4 b)$. We did not observe significant differences in IgA+ plasma cells or stool IgA levels between the mice gavaged with $\mathrm{CD}$ or non-CD stool (online supplementary figure S9).

We also observed a reduced frequency of regulatory $\mathrm{T}$ cells $\left(\mathrm{T}_{\mathrm{REG}}\right.$, live $\mathrm{CD} 45^{+} \mathrm{CD} 3{ }^{+} \mathrm{CD} 8{ }^{-} \mathrm{CD} 4{ }^{+}$Foxp $3{ }^{+}$cells, online supplementary figures 7 and 8 in the colonic LP $(p<0.005)$ and MLN $(p<0.05)$ of mice inoculated with CD maternal stool compared with control maternal stool; no differences were found between the mice inoculated with the infant stool by maternal CD diagnosis (figure 4C).

\section{DISCUSSION}

Our findings show that the microbiome of mothers with IBD was characterised by a lower $\alpha$-diversity during the first and second trimester of pregnancy and a different $\beta$-diversity during each of the three trimesters compared with control mothers (figure $2 \mathrm{~A}, \mathrm{~B})$. The differences were driven by a depletion in the relative abundance of Bacteroidetes and an increase in the relative abundance of Proteobacteria (figure 2C). Importantly, we observed that the offspring of IBD mothers presented with a reduced bacterial diversity as early as by the end of the first week of life and demonstrated differences in the overall gut bacterial composition persisting for up to 3 months of life compared with control babies (figure $3 \mathrm{~A}, \mathrm{~B}$ ). These data are in line with previous studies reporting the impact of maternal diseases, such as obesity, ${ }^{27}$ eczema, ${ }^{28} \mathrm{HIV}$ status ${ }^{29}$ and gestational diabetes, ${ }^{30}$ on the offspring's microbiome. In our study, early-life microbial alterations were driven by enrichment in Gammaproteobacteria and a reduction in Bifidobacterium at each time point (figure 3C-E), mirroring some of the patterns observed in their mothers, as well as in adult and childhood-onset IBD patients. ${ }^{3132}$ Bifidobacteria are among the first colonisers of the infant gut and are believed to promote health benefits for the host, stimulating maturation of the immune system and contributing to defence against enteropathogens. ${ }^{33}$ Bifidobacteria species have been shown to protect against development of colitis, ${ }^{34}$ and in patients with IBD, multistrain Bifidobacteriumcontaining probiotics have been shown to be effective in specific settings. ${ }^{35}$ However, Proteobacteria are often associated with intestinal inflammation ${ }^{36}$ and have been consistently linked to IBD. $^{23}$

While maternal IBD status was the major and most consistent factor associated with baby's microbiome composition at each time point, other factors, such as mode of delivery, feeding behaviour, exposure to antibiotics and preterm birth, were also found to affect the overall microbial composition. C-section and exposure to antibiotics during pregnancy and early life have been shown to increase the risk of IBD later in life, ${ }^{3738}$ while breastfeeding in infancy has been shown to be protective against the development of IBD. ${ }^{8-1139}$ Moreover, when analysing the most differentially prevalent taxa between babies by maternal IBD status over time, exclusive breastfeeding was independently associated with higher relative abundance of Bifidobacterium, which is consistent with prior reports. ${ }^{40}$

Pregnancy and early life are a critical period during which contact with microbes and specific microbe-associated metabolites are essential for the priming of the immune system development and maturation. ${ }^{41}$ Therefore, to assess functional consequences of the observed IBD-associated dysbiosis, we inoculated GFM, with the stool of pregnant women with and without $\mathrm{CD}$ and their babies. ${ }^{42}$ Significant differences were observed in the murine microbiome composition based 
(a) Class-switched memory B cell

Colonic lamina propria

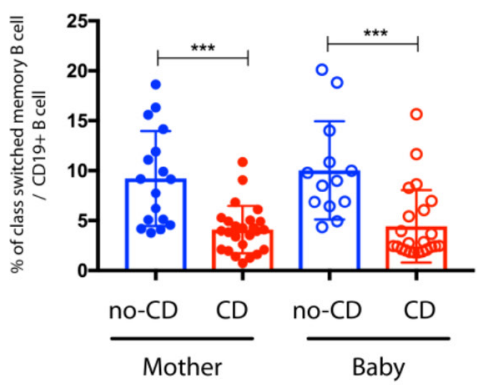

(b) IgA+ class switched memory B cell

Colonic lamina propria

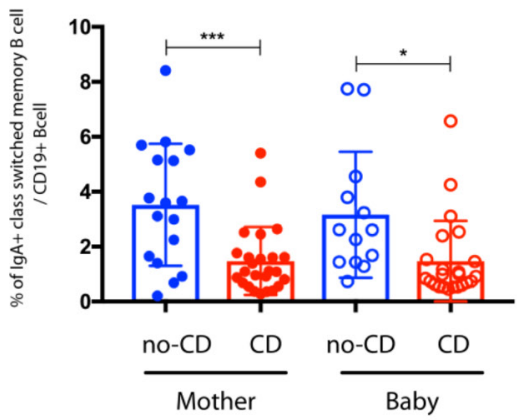

(c) Regulatory Tcell

Colonic lamina propria

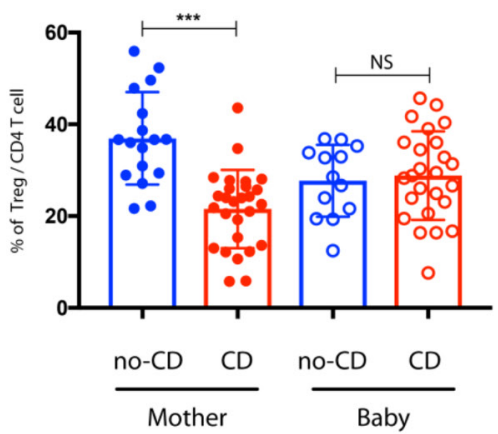

Lymph node

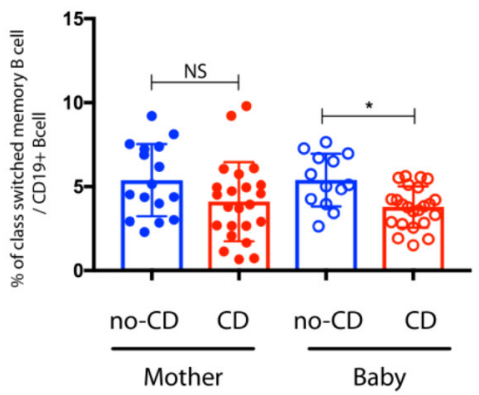

Lymph node

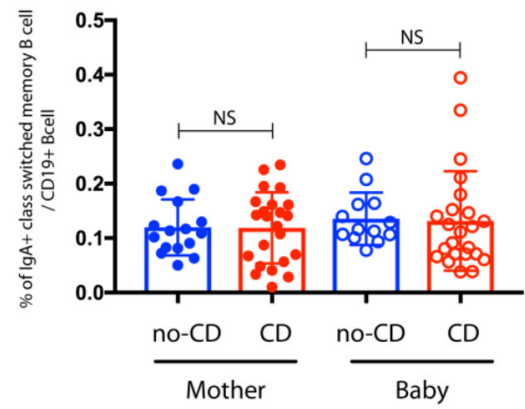

Lymph node

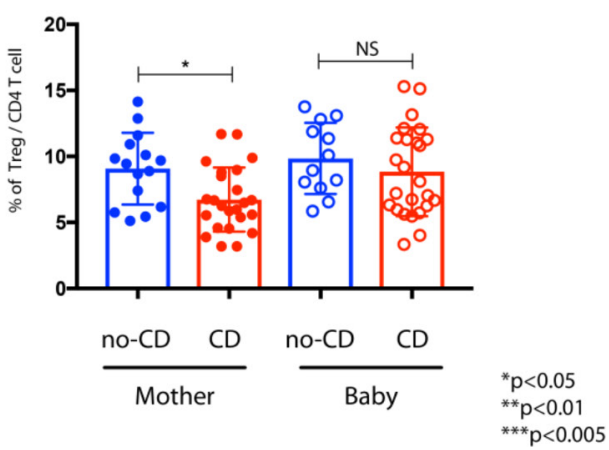

Figure 4 Germ-free mice inoculated with the stool of mothers with $C D$ and their 3-month-old babies have significant abnormalities in the adaptive immune cells compared with mice inoculated with stool from non-CD controls. (A) Class-switched memory B cells (aqua ${ }^{-}$, $\mathrm{CD}_{4} 5^{+} \mathrm{CD} 19^{+} \mathrm{CD}_{138^{-}} \mathrm{IgD}^{-} \mathrm{IgM}^{-}$) isolated from the colonic lamina propria and mesenteric lymph node (MLN) of GFM, which were inoculated with the stool of non-CD mother, CD mother or their 3-month-old babies (non-CD baby or CD baby). Cumulative data showing the percentage of classswitched B cell among live CD19+ ${ }^{+} 138^{-}$B cells in the indicated groups of colonic lamina propria or MLN. (B) Cumulative data showing the

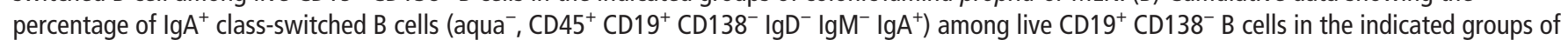
colonic lamina propria or MLN. (C) Cumulative data showing the percentage of regulatory $\mathrm{T}_{\text {cells }}\left(\mathrm{T}_{\mathrm{REG}}\right)$ (aqua ${ }^{-}, \mathrm{CD} 5^{+} \mathrm{CD}^{+} \mathrm{CD} 8^{-} \mathrm{CD} 4^{+} \mathrm{FoxP3} 3^{+}$) among live $\mathrm{CD}_{4} 5^{+} \mathrm{CD} 3^{+} \mathrm{CD} 8^{-} \mathrm{CD} 4^{+}$cells in the indicated groups of colonic lamina propria or $\mathrm{MLN} .{ }^{*} \mathrm{P}<0.05$, ** $\mathrm{p}<0.01,{ }^{* *} \mathrm{*}<<0.005$. $\mathrm{CD}, \mathrm{Crohn}$ 's disease; NS, not significant.

on maternal CD status (online supplementary figure S5. Interestingly, exposure of GFM to the microbiome of pregnant CD donors at T3 resulted in disruptions of critical homeostatic elements of the immune system with a lower proportion of FoxP $3{ }^{+} \mathrm{T}_{\mathrm{REG}}$, and IgA-expressing class-switched memory B cells in the colonic LP, and a reduction of FoxP $3^{+} \mathrm{T}_{\mathrm{REG}}$ also in MLN compared with the exposure to control pregnant women's stool (figure 4). Furthermore, inoculation with the stool from 3-month-old babies born to mothers with CD also led to a lower proportion of class-switched memory B cells in the colonic LP and MLN, and homeostatic IgA + B cells in the colonic LP. $\mathrm{T}_{\mathrm{REG}}$ have a well-established role in the suppression of systemic and mucosal immune activation and in the regulation of intestinal inflammation. ${ }^{43}$ Similarly, IgA, the predominant antibody at mucosal surfaces, is of critical importance to mucosal homeostasis. IgA is associated with downregulation of proinflammatory epitopes on commensal bacteria, ${ }^{44}$ secretion of a biofilm that favours the growth of commensals, ${ }^{45}$ direction of luminal 
bacteria to $\mathrm{M}$ cells,${ }^{46}$ maturation of dendritic cells,${ }^{47}$ production of interleukin- $10^{48}$ and FcoRI-mediated suppression of immune responses. ${ }^{49}$ It has been suggested that patients with IBD have an impaired production of polymeric $\operatorname{IgA}$ at the mucosal surfaces, ${ }^{50} 51$ and a significant reduction in peripheral switched memory IgD-CD27+ B cells has been described in the blood of 1 -year-old infants born to IBD mothers treated with combination therapy. ${ }^{52}$ Previous reports have shown that intestinal bacterial colonisation pattern may affect B cell maturation in humans and that early gut microbiota might promote this maturation. ${ }^{53}$ Together, reduced frequencies of $\mathrm{T}_{\mathrm{REG}}$ and $\mathrm{IgA}+\mathrm{B}$ cell subsets in the colon of GFM inoculated with IBD-associated stools may indicate reduced frequencies of two critical homeostatic elements of the mucosal immune system that are driven by IBDassociated dysbiosis.

Our data provide a potential link between early life exposures, microbiome and future risk of IBD, underscoring the potential consequences of the aberrant formation of early-life microbiome during the sensitive time window of the immune system development. Of note, low bacterial diversity in early life has been shown to precede development of diseases such as asthma ${ }^{54}$ or allergies in childhood. ${ }^{55}$ Considering the central role of gut microbiome in IBD pathogenesis, we speculate that the observed patterns may, at least in part, help to explain the residual familiar risk of IBD in the offspring beyond the established shared genetic risk.

The strengths of our study include a unique prospective cohort of pregnant women with and without IBD and their babies, with serial sample collection and exposure data. For the first time, we were able to investigate the role of the major maternal and early life exposures on the rapid changes in the developing gut microbiome. Our study's limitations include inability to correct for all potential confounders, such as IBD type, disease location, activity, complications, medications and other IBD-related variables that may impact the microbiome. Small numbers of babies exposed to antibiotics and exclusive formula feeding prevented us from further assessing the impact and interaction of these environmental exposures, which are known to affect infants' gut colonisation. Moreover, we did not have access to blood from infants, and therefore, we resorted to a validated model system (GFM) to anticipate the immune-related consequences of the imbalanced microbiome. ${ }^{56}$ Also, we are reporting on changes in gut microbiome and immunological profiles induced by $\mathrm{CD}$-associated donor stools in healthy mice. Yet, the impact of CD-associated dysbiosis on susceptibility to colitis cannot be inferred, neither can the underlying pathophysiological mechanisms driving the observed effects be determined. Future studies are warranted to test our findings in murine models of mucosal inflammation. Finally, although we see clear and reproducible changes within the adaptive immune system, we could not identify similar changes in innate immune cells. Lack of sensitivity, absence of inflammation or changes within alternative cellular subsets are among the possible explanations.

In summary, using a prospective cohort, we detected a significant association of maternal IBD with changes in microbiome composition during pregnancy and in the offspring. Exposure to the microbiome of pregnant patients with CD or their babies led to the development of an imbalanced immune system lacking critical homeostatic elements in a humanised experimental model. Understanding the critical events shaping the microbiome of mothers with IBD and their offspring can ultimately help to develop new strategies aiming at disease prediction and prevention.

\section{Author affiliations}

${ }^{1}$ Dr. Henry D. Janowitz Division of Gastroenterology, Icahn School of Medicine at Mount Sinai, New York City, New York, USA

${ }^{2}$ Department of Gastroenterology, Hospital Beatriz Angelo, Loures, Portugal

${ }^{3}$ Department of Genetics and Genomic Sciences, Icahn School of Medicine at Mount Sinai, New York City, New York, USA

${ }^{4}$ The Precision Immunology Institute, Icahn School of Medicine at Mount Sinai, New York City, New York, USA

${ }^{5}$ Department of Health Evidence and Policy, Icahn School of Medicine at Mount Sinai, New York City, New York, USA

${ }^{6}$ Department of Gastroenterology and Hepatology, Meander Medical Center, Amersfoort, The Netherlands

${ }^{7}$ Department of Gastroenterology, Centre Hospitalier de L'Universite de Montreal, Montreal, Quebec, Canada

${ }^{8}$ Department of Thoracic Surgery, Jiangsu Cancer Hospital, Jiangsu Institute of Cancer Research, Nanjing Medical University Affiliated Cancer Hospital, Naijing, China

${ }^{9}$ The Precision Immunology Institute Icahn School of Medicine at Mount Sinai, New York City, New York, USA

${ }^{10}$ Department of Obstetrics, Gynecology and Reproductive Science, Icahn School of Medicine at Mount Sinai, New York City, New York, USA

${ }^{11}$ Department of Gastroenterology, Icahn School of Medicine at Mount Sinai, New York City, New York, USA

${ }^{12}$ Department of Pediatrics, Icahn School of Medicine at Mount Sinai, New York City, New York, USA

Acknowledgements We would like to thank our study participants for their invaluable contribution to this project.

Contributors JT and JH contributed equally. JT, J-FC, IP, JH and BJ conceived and designed the study. CE, NN, LT, QM, EM, C-LC, AK, JG, PL, HL, JS, JC-D and MD managed subject recruitment, data and sample collection and sample processing. GB, IM, JF, LT and AS designed and conducted germ-free mice (GFM) experiments. AS, MU and SM designed and conducted immune profiling experiments. JH, JT, JCC, AS and RH generated and analysed data. JT, J-FC, IP, SM and JH wrote the manuscript. All authors read the manuscript and provided critical comments.

Funding This work was funded by the International Organization for the Study of Inflammatory Bowel Disease (to IP and J-FC.), The Crohn's and Colitis Foundation (to IP, JT, JCC and J-FC), and the Kenneth Rainin Foundation (to IP, J-FC, JF and SM). We would like to express our gratitude for Dr Jixin Dai and Ms Yi Liu's generosity in supporting the MECONIUM study.

Competing interests JT received lecture fees from Takeda and Abbvie. JJF is a consultant for Janssen Research \& Development and a member of the Scientific Advisory Board of Vedanta Biosciences. JFC reports receiving research grants from AbbVie, Janssen Pharmaceuticals and Takeda; receiving payment for lectures from AbbVie, Amgen, Allergan, Inc. Ferring Pharmaceuticals, Shire, and Takeda; receiving consulting fees from AbbVie, Amgen, Arena Pharmaceuticals, Boehringer Ingelheim Celgene Corporation, Celltrion, Eli Lilly, Enterome, Ferring Pharmaceuticals,

Genentech, Janssen Pharmaceuticals, Landos, Ipsen, Medimmune, Merck,Novartis,

Pfizer, Shire, Takeda, Tigenix and holding stock options in Intestinal Biotech Development and Genfit.

Patient consent for publication Parental/guardian consent obtained

Ethics approval All experiments were performed using protocols approved by the local animal ethics committee.

Provenance and peer review Not commissioned; externally peer reviewed.

\section{ORCID iDs}

Joana Torres http://orcid.org/0000-0003-2895-5821

Mathieu Uzzan http://orcid.org/0000-0002-7761-2879

\section{REFERENCES}

1 Torres J, Mehandru S, Colombel J-F, et al. Crohn's disease. The Lancet 2017;389:1741-55.

2 Ungaro R, Mehandru S, Allen PB, et al. Ulcerative colitis. Lancet 2017;389:1756-70.

3 Zelinkova Z, Stokkers PC, van der Linde K, et al. Maternal imprinting and female predominance in familial Crohn's disease. J Crohns Colitis 2012;6:771-6.

4 Akolkar PN, Gulwani-Akolkar B, Heresbach D, et al. Differences in risk of Crohn's disease in offspring of mothers and fathers with inflammatory bowel disease. Am J Gastroenterol 1997;92:2241-4.

5 Koren $\mathrm{O}$, Goodrich JK, Cullender TC, et al. Host remodeling of the gut microbiome and metabolic changes during pregnancy. Cell 2012;150:470-80.

6 Ferretti P, Pasolli E, Tett A, et al. Mother-to-infant microbial transmission from different body sites shapes the developing infant gut microbiome. Cell Host Microbe 2018:24:133-45

7 Gomez de Agüero M, Ganal-Vonarburg SC, Fuhrer T, et al. The maternal microbiota drives early postnatal innate immune development. Science 2016;351:1296-302. 
8 Benchimol El, Kaplan GG, Otley AR, et al. Rural and urban residence during early life is associated with risk of inflammatory bowel disease: a population-based inception and birth cohort study. Am J Gastroenterol 2017;112:1412-22.

9 Kronman MP, Zaoutis TE, Haynes K, et al. Antibiotic exposure and IBD development among children: a population-based cohort study. Pediatrics 2012;130:e794-e803.

10 Sevelsted A, Bisgaard $\mathrm{H}$. Neonatal size in term children is associated with asthma at age 7, but not with atopic dermatitis or allergic sensitization. Allergy 2012;67:670-5.

11 Xu L, Lochhead P, Ko Y, et al. Systematic review with meta-analysis: breastfeeding and the risk of Crohn's disease and ulcerative colitis. Aliment Pharmacol Ther 2017:46:780-9.

12 Mahowald MA, Rey FE, Seedorf $\mathrm{H}$, et al. Characterizing a model human gut microbiota composed of members of its two dominant bacterial phyla. Proc Natl Acad Sci U S A 2009;106:5859-64.

13 Best WR. Predicting the Crohn's disease activity index from the Harvey-Bradshaw Index. Inflamm Bowel Dis 2006:12:304-10.

14 Schroeder KW, Tremaine WJ, Ilstrup DM. Coated oral 5-aminosalicylic acid therapy for mildly to moderately active ulcerative colitis. A randomized study. N Engl J Med 1987;317:1625-9.

15 Harris PA, Taylor R, Thielke R, et al. Research electronic data capture (REDCap)--a metadata-driven methodology and workflow process for providing translational research informatics support. J Biomed Inform 2009;42:377-81.

16 Torres J, Bao X, Goel A, et al. The features of mucosa-associated microbiota in primary sclerosing cholangitis. Aliment Pharmacol Ther 2016;43:790-801.

17 Masella AP, Bartram AK, Truszkowski JM, et al. PANDAseq: paired-end assembler for illumina sequences. BMC Bioinformatics 2012;13:31.

18 Caporaso JG, Kuczynski J, Stombaugh J, et al. QIIME allows analysis of highthroughput community sequencing data. Nat Methods 2010;7:335-6.

19 Faith DP, Baker AM, diversity P. PD) and biodiversity conservation: some bioinformatics challenges. Evol Bioinform Online 2007;2:121-8.

20 Lozupone C, Knight R. UniFrac: a new phylogenetic method for comparing microbial communities. Appl Environ Microbiol 2005;71:8228-35.

21 Mason NWH, de Bello F, Doležal J, et al. Niche overlap reveals the effects of competition, disturbance and contrasting assembly processes in experimental grassland communities. J Ecol 2011;99:788-96.

22 Segata $\mathrm{N}$, Izard J, Waldron L, et al. Metagenomic biomarker discovery and explanation. Genome Biol 2011;12:R60.

23 Morgan XC, Tickle TL, Sokol H, et al. Dysfunction of the intestinal microbiome in inflammatory bowel disease and treatment. Genome Biol 2012;13:R79.

24 Faith JJ, Ahern PP, Ridaura VK, et al. Identifying gut microbe-host phenotype relationships using combinatorial communities in gnotobiotic mice. Sci Trans/ Med 2014:6:220ra11.

25 Turnbaugh PJ, Ridaura VK, Faith JJ, et al. The effect of diet on the human gut microbiome: a metagenomic analysis in humanized gnotobiotic mice. Sci Trans/ Med 2009;1:6ra14.

26 Ruane $\mathrm{D}$, Chorny $\mathrm{A}$, Lee $\mathrm{H}$, et al. Microbiota regulate the ability of lung dendritic cells to induce IgA class-switch recombination and generate protective gastrointestinal immune responses. J Exp Med 2016;213:53-73.

27 Collado MC, Isolauri E, Laitinen $\mathrm{K}$, et al. Effect of mother's weight on infant's microbiota acquisition, composition, and activity during early infancy: a prospective follow-up study initiated in early pregnancy. Am J Clin Nutr 2010;92:1023-30.

28 Gosalbes MJ, Llop S, Vallès Y, et al. Meconium microbiota types dominated by lactic acid or enteric bacteria are differentially associated with maternal eczema and respiratory problems in infants. Clin Exp Allergy 2013;43:198-211.

29 Bender JM, Li F, Martelly S, et al. Maternal HIV infection influences the microbiome of HIV-uninfected infants. Sci Trans/ Med 2016;349:ra100.

$30 \mathrm{Hu}$ J, Nomura Y, Bashir A, et al. Diversified microbiota of meconium is affected by maternal diabetes status. PLoS One 2013;8:e78257.

31 Kostic AD, Xavier RJ, Gevers D. The microbiome in inflammatory bowel disease: current status and the future ahead. Gastroenterology 2014;146:1489-99.
32 Gevers D, Kugathasan S, Denson LA, et al. The treatment-naive microbiome in newonset Crohn's disease. Cell Host Microbe 2014:15:382-92.

33 Hidalgo-Cantabrana C, Delgado S, Ruiz L, et al. Bifidobacteria and their healthpromoting effects. Microbio/ Spectr 2017;5.

34 Lee IA, Bae EA, Lee JH, et al. Bifidobacterium longum HY8004 attenuates TNBSinduced colitis by inhibiting lipid peroxidation in mice. Inflamm Res 2010;59:359-68.

35 Harbord M, Eliakim R, Bettenworth D, et al. Third European Evidence-based Consensus on Diagnosis and Management of Ulcerative Colitis. Part 2: Current Management. J Crohns Colitis 2017;11:769-84.

36 Shin NR, Whon TW, Bae JW. Proteobacteria: microbial signature of dysbiosis in gut microbiota. Trends Biotechnol 2015:33:496-503.

37 Örtqvist AK, Lundholm C, Halfvarson J, et al. Fetal and early life antibiotics exposure and very early onset inflammatory bowel disease: a population-based study. Gut 2019;68:218-25.

38 Sevelsted A, Stokholm J, Bønnelykke K, et al. Cesarean section and chronic immune disorders. Pediatrics 2015;135:e92-e98.

39 Benchimol El, Mack DR, Guttmann A, et al. Inflammatory bowel disease in immigrants to Canada and their children: a population-based cohort study. Am J Gastroenterol 2015;110:553-63.

40 Grönlund MM, Gueimonde M, Laitinen K, et al. Maternal breast-milk and intestinal bifidobacteria guide the compositional development of the Bifidobacterium microbiota in infants at risk of allergic disease. Clin Exp Allergy 2007;37:1764-72.

41 Laforest-Lapointe I, Arrieta MC. Patterns of early-life gut microbial colonization during human immune development: an ecological perspective. Front Immunol 2017;8:788.

42 Round JL, Mazmanian SK. The gut microbiota shapes intestinal immune responses during health and disease. Nat Rev Immunol 2009;9:313-23.

43 Izcue A, Coombes JL, Powrie F. Regulatory T cells suppress systemic and mucosal immune activation to control intestinal inflammation. Immunol Rev 2006;212:256-71.

44 Peterson DA, McNulty NP, Guruge JL, et al. IgA response to symbiotic bacteria as a mediator of gut homeostasis. Cell Host Microbe 2007;2:328-39.

45 Bollinger RR, Everett ML, Wahl SD, et al. Secretory IgA and mucin-mediated biofilm formation by environmental strains of Escherichia coli: role of type 1 pili. $\mathrm{Mol}$ Immuno 2006:43:378-87

46 Corr SC, Gahan CC, Hill C. M-cells: origin, morphology and role in mucosal immunity and microbial pathogenesis. FEMS Immunol Med Microbiol 2008;52:2-12.

47 Geissmann F, Launay P, Pasquier B, et al. A subset of human dendritic cells expresses IgA Fc receptor (CD89), which mediates internalization and activation upon cross linking by IgA complexes. J Immuno/ 2001;166:346-52.

48 Pilette C, Detry B, Guisset A, et al. Induction of interleukin-10 expression through Fcalpha receptor in human monocytes and monocyte-derived dendritic cells: role of p38 MAPKinase. Immunol Cell Biol 2010;88:486-93.

49 Phalipon A, Corthésy B. Novel functions of the polymeric lg receptor: well beyond transport of immunoglobulins. Trends Immunol 2003;24:55-8

50 Uzzan M, Colombel JF, Cerutti A, et al. B Cell-Activating Factor (BAFF)-Targeted B Cell therapies in inflammatory bowel diseases. Dig Dis Sci 2016;61:3407-24.

51 Takiguchi H, Endo S, Omagari D, et al. Reduced production of polymeric immunoglobulin receptor in murine dextran sodium sulfate-induced colitis. J Ora/ $\mathrm{SCl}$ 2012;54:23-32.

52 Kattah M, Milush J, Burt T, et al. Multiparameter flow cytometry reveals differences in b cells subsets among infants born to mothers being treated for IBD. Gastroenterology 2017:152:S86

53 Lundell AC, Björnsson V, Ljung A, et al. Infant B cell memory differentiation and early gut bacterial colonization. J Immunol 2012;188:4315-22.

54 Abrahamsson TR, Jakobsson $\mathrm{HE}$, Andersson AF, et al. Low gut microbiota diversity in early infancy precedes asthma at school age. Clin Exp Allergy 2014;44:842-50

55 Azad MB, Konya T, Guttman DS, et al. Infant gut microbiota and food sensitization: associations in the first year of life. Clin Exp Allergy 2015;45:632-43.

56 Arrieta MC, Walter J, Finlay BB. Human microbiota-associated mice: a model with challenges. Cell Host Microbe 2016;19:575-8. 